\title{
INVESTIGACIÓN
}

Recibido: 03/05/2021 --- Aceptado: 03/12/2021 --- Publicado: 03/01/2022

\section{LAS COMPETENCIAS DIGITALES EN PERSONAS MAYORES: DE AMENAZA A OPORTUNIDAD}

Digital competencies in older adults: from threat to opportunity

Eva Bunbury Bustillo. Universidad de Zaragoza. España ebunbury1@alumno.uned.es

Ricardo Pérez Calle. Universidad de Zaragoza. España rdperez@unizar.es

Sara Osuna-Acedo. Universidad Nacional de Educación a Distancia (UNED) sosuna@edu.uned.es

Cómo citar el artículo:

Bunbury Bustillo, E., Pérez Calle, R., y Osuna-Acedo, S. (2022). Las Competencias Digitales en personas mayores: de amenaza a oportunidad. Vivat Academia. Revista de Comunicación, 155, 173-195. http://doi.org/10.15178/va.2022.155.e1383

\section{RESUMEN}

El envejecimiento poblacional es un fenómeno social presente en la mayoría de los países avanzados, lo que supone para los distintos gobiernos, organizaciones y sociedades tener que afrontar nuevos retos. Prolongar la autonomía, la salud y la calidad de vida de las personas mayores, fomentando a su vez un envejecimiento activo, se constituye en una prioridad en este sentido. Para el logro de estos objetivos, surge como una necesidad imperiosa la reducción de la brecha digital presente de forma común en el colectivo de las personas mayores. A través de la presentación de dos proyectos reales, llevados a cabo en la ciudad de Zaragoza, con la colaboración activa entre colectivos de personas de mayor edad, se presentan evidencias de la situación de vulnerabilidad digital en la que se encuentran las personas mayores, así como de su predisposición y voluntad para mejorar su capacitación en el uso de las nuevas tecnologías. El análisis de los resultados obtenidos en ambos proyectos nos lleva a concluir que el potencial que para las personas mayores ofrece el uso habitual de las TIC es enorme, con una larga lista de oportunidades existentes para el beneficio de este colectivo que deben ser aprovechadas. 
Bunbury Bustillo, E., Pérez Calle, R., y Osuna-Acedo, S.

Las Competencias Digitales en personas mayores: de amenaza a oportunidad

PALABRAS CLAVE: TRIC - Brecha digital - Envejecimiento poblacional Envejecimiento activo - Ciudades saludables - Capacitación digital Empoderamiento - Grupos focales.

\begin{abstract}
Population aging is a social phenomenon present in most advanced countries, which means that governments, organizations and societies face new challenges. Prolonging the autonomy, health and quality of life of the elderly, while promoting active aging, is a priority in this regard. In order to achieve these objectives, the reduction of the digital divide, which is common among the elderly, is an urgent need. Through the presentation of two real projects, carried out in the city of Zaragoza, with the active collaboration between groups of older people, evidence is presented of the situation of digital vulnerability in which older people find themselves, as well as their predisposition and willingness to improve their skills in the use of new technologies. The analysis of the results obtained in both projects leads us to conclude that the potential offered by the regular use of ICTs for the elderly is enormous, with a long list of existing opportunities for the benefit of this group that should be taken into full consideration.
\end{abstract}

KEYWORDS: ICT - Digital divide - Population aging - Active aging - Healthy cities Digital empowerment - Empowerment - Focus groups.

\title{
HABILIDADES DIGITAIS EM PESSOAS IDOSAS: DA AMEAÇA À OPORTUNIDADE
}

\section{RESUMO}

O envelhecimento populacional é um fenômeno social presente na maioria dos países avançados, o que significa que diferentes governos, organizações e sociedades têm que enfrentar novos desafios. Prolongar a autonomia, saúde e qualidade de vida dos idosos, ao mesmo tempo que se promove o envelhecimento ativo, é uma prioridade neste quesito. Para atingir esses objetivos, a redução da exclusão digital comumente presente no grupo de idosos surge como uma necessidade imperiosa. Através da apresentação de dois projetos reais, realizados na cidade de Saragoça, com a colaboração ativa entre grupos de idosos, são apresentadas evidências da situação de vulnerabilidade digital em que se encontram os idosos, bem como a sua predisposição e disposição para aprimorar sua capacitação no uso de novas tecnologias. A análise dos resultados obtidos em ambos os projetos leva-nos a concluir que o potencial que o uso habitual das TIC oferece aos idosos é enorme, existindo uma longa lista de oportunidades em benefício deste grupo que devem ser exploradas.

PALAVRAS-CHAVE: TRIC - Cisão digital - Envelhecimento populacional Envelhecimento ativo - Cidades saudáveis - Treinamento digital - Empoderamento Grupos focais. 
Bunbury Bustillo, E., Pérez Calle, R., y Osuna-Acedo, S.

Las Competencias Digitales en personas mayores: de amenaza a oportunidad

\section{INTRODUCCIÓN}

El envejecimiento de la población es una realidad social presente en las sociedades avanzadas, que siendo un fenómeno global es especialmente acusado en determinados países como España (Domènech, 2019). El aumento de la esperanza de vida, conjugado con una constante disminución de la tasa de natalidad desde el final del denominado baby boom en los años setenta del pasado siglo, ha provocado que la pirámide poblacional haya ido siguiendo un paulatino proceso de inversión a lo largo de las últimas décadas (Díaz \& García, 2018). En España (Ministerio de Sanidad, 2020) la esperanza de vida alcanza actualmente los 83,4 años- con un aumento de más de cuatro años desde el año 2000- y el porcentaje de población por encima de los 65 años se sitúa en el 19,7\% del total. Respecto al índice de envejecimiento ${ }^{1}$, se encuentra en el $120 \%$.

El fenómeno social del envejecimiento es uno de acontecimientos con un mayor impacto esperado en el futuro a corto y medio plazo (Conde-Ruíz \& González, 2021). Implica, para los gobiernos y sociedades, el tener que prepararse para afrontar nuevos retos. Así, a los problemas clásicos relacionados con la asistencia sanitaria o la dependencia- cuya intensidad e incidencia se irán a su vez incrementando- se han ido e irán añadiendo otros asuntos relacionados con los fenómenos de la soledad y el aislamiento entre las personas mayores. Situaciones personales que conjugan edad avanzada, ausencia de una red familiar de apoyo y en ocasiones precariedad económica pueden no incidir de forma directa e inmediata en la salud y en la capacidad de autonomía de las personas, pero generan con el tiempo aislamiento social y consecuencias negativas para la salud y para la capacidad cognitiva (Arranz et al., 2009; Puig Llobet et al., 2009).

De esta forma, la realidad social del envejecimiento ha de ser abordada desde un enfoque amplio que considere la amplitud y variedad de factores implicados. Según se pronuncia la Organización Mundial de la Salud (OMS) en su sección Aging and lifecourse, "la salud y el bienestar están determinados no sólo por nuestros genes y características personales, sino también por el entorno físico y social en el que vivimos" (Organización Mundial de la Salud, s.f.). Con ello, los entornos en su sentido más amplio se convierten en parte del problema y de la solución, desempeñando un papel importante a la hora de determinar la capacidad física y mental a lo largo de la vida de una persona hasta alcanzar una edad avanzada (Osorio et al., 2010), así como la forma en que se adapta a la pérdida de funciones y a otras formas de adversidad que se pueden experimentar en diferentes etapas de la vida y en particular en los últimos años. Tanto las personas mayores como los entornos en los que viven son diversos, dinámicos y cambiantes. Al interactuar entre sí, tienen un increíble potencial para permitir o limitar un envejecimiento saludable (Petretto et al., 2016; Triadó \& Villar, 2008).

\footnotetext{
${ }^{1}$ Cociente entre la población mayor de 65 años y la menor de 16 años.
} 
A nivel institucional supranacional, la mejora de calidad de vida de los mayores es uno de los pilares de acción de distintos programas sociales del ámbito público. Así, desde 1995 el Comité Europeo de Cohesión Social del Consejo de Europa (CECS) coordina, guía y estimula la cooperación entre los Estados miembros en relación a las acciones centradas en las personas de la tercera edad y especialmente el de las dependientes. En concreto, el Grupo de Expertos en la Mejora de la Calidad de Vida de las Personas Mayores Dependientes (CS-QV) desarrolla su labor apoyándose en las ideas expuestas por organismos europeos e internacionales relacionados con la calidad de vida de las personas de la tercera edad, con el objetivo de proponer y fomentar políticas y recursos para optimizar las condiciones de vida de este segmento poblacional, manteniendo su calidad de vida y autonomía el máximo tiempo posible. Sus acciones se centran en aspectos como (O'Shea, 2003):

- Sensibilizar a la población sobre los problemas del envejecimiento y la dependencia.

- Promover medidas para reducir las desigualdades en las condiciones de vida de las personas mayores.

- Promover la integración social de las personas mayores dependientes.

- Promover la difusión de información y orientación sobre ayudas y diferentes servicios existentes para las personas mayores.

- Fomentar y potenciar las diferentes formas de solidaridad (familia, vecinos, voluntarios).

- Mantener los desarrollos nacionales de legislación y prácticas en el ámbito de la tercera edad y la dependencia.

A nivel mundial, en la actualidad existen ciudades y comunidades que trabajan con el objetivo de ser más amigables con las personas de la tercera edad (Organización Mundial de la Salud, 2007). La Red Mundial de la OMS está formada por más de 830 ciudades y comunidades de 41 países, que trabajan para mejorar sus entornos físicos y sociales y convertirse en mejores lugares para envejecer (Zamarro, 2017). La creación de estos entornos respetuosos con la edad requiere de la adopción de medidas en muchos sectores: salud, atención a la dependencia, transporte, vivienda, trabajo, protección social, información y comunicación, etc. Requiere del trabajo y de la cooperación de muchos agentes- gobiernos, proveedores de servicios, sociedad civil, organizaciones no gubernamentales- y además de la implicación de las propias personas mayores y de sus familias y amistades. También exige la actuación de múltiples niveles de la administración, desde internacional hasta local. Este último nivel adquiere un papel protagonista activo en el éxito de las distintas acciones llevadas a cabo, debido a una cualidad clave como es la de su proximidad al usuario final. Las ciudades en el siglo XXI se van constituyendo así en actores de enorme influencia sobre la calidad y forma de vida de las personas, adquiriendo nuevos 
adjetivos como inteligentes, interconectadas, sostenibles o amigables (Caragliu et al., 2011; Rodríguez-Porrero \& Gil-González, 2014; Telefónica, 2011).

De forma común, sobre las distintas acciones y líneas de trabajo de la Red Mundial de la OMS se pueden identificar los siguientes enfoques sobre las distintas partes interesadas (Fernández-Ballesteros, 2011; Fernández-Ballesteros et al., 2011; Havighurst, 1963):

- Combatir la discriminación por razón de edad;

- permitir la autonomía;

- apoyar el envejecimiento saludable en todas las políticas a todos los niveles.

En concreto, la OMS fomenta la creación de entornos favorables con la edad, a través de (Organización Mundial de la Salud, s.f., 2007; Zamarro Cuesta, 2017):

- la recopilación de orientaciones basadas en la evidencia sobre los entornos favorables con la edad;

- plataformas de información para compartir información y experiencias; y

- la generación y desarrollo de la Red Mundial de Ciudades y Comunidades Amigables con las Personas Mayores de la OMS.

En el caso concreto de las personas mayores, existen tres elementos que deben tenerse en cuenta en la realización de un adecuado análisis sobre la información y el diseño de las políticas dirigidas a lograr un entorno adaptado a sus necesidades (Fernández-Ballesteros, 2011; Fernández del Moral, 2012; Marta-Lazo y Gabelas, 2016; Pérez-Calle et al., 2020). En primer lugar, los contenidos. Las personas mayores tienen que disponer de información adecuada a sus necesidades. En segundo lugar, adaptación de esta información y de los canales de comunicación a través de los que acceden a la misma. En tercer lugar, la necesidad de que participen en la sociedad de la información, superando la llamada brecha digital.

Respecto a los contenidos, disponer de información suficiente y accesible supone para los mayores acrecentar las posibilidades de mantener el contacto con otras personas, participar en eventos interesantes para ellos, administrar su vida y atender sus necesidades personales. El desarrollo de las actividades diarias y habituales por las personas mayores y la satisfacción de sus necesidades en los diferentes órdenes precisa de la información necesaria. La participación social, por ejemplo, se ve facilitada claramente si se dispone de información adecuada para poder tomar parte en las actividades programadas.

Respecto a la adaptación de la información y el uso de los canales de comunicación más adecuados, es importante reseñar que la recepción de la información se convierte en un derecho vinculado a disfrutar de una calidad de vida óptima y que 
la garantía de derechos se entiende como el definir los contenidos de información precisos y el establecimiento de los canales de comunicación adecuados. El hecho fundamental es que la información sea fácilmente accesible a las personas mayores en todos sus contenidos, asumiendo que de acuerdo a la necesidad a cubrir estos variarán. La cultura es un ejemplo, pero hay otros aspectos en los que la información se convierte en un derecho y un servicio para las personas mayores. La Unión Democrática de Pensionistas y Jubilados de España (2019) realiza un dimensionamiento de esta problemática, destacando que más del $15 \%$ de las personas mayores no se consideran con conocimientos e información suficiente para realizar todo lo que necesitan para vivir como desearían. El porcentaje sobrepasa el $20 \%$ entre los que tienen un nivel inferior de estudios, el 21,8\%; entre quienes tienen una capacidad de gasto insuficiente, el 21,2\%; o entre quienes viven en el ámbito rural, el 23,6\%.

Finalmente, la brecha digital se constituye en un factor clave en el proceso. Su eliminación debe fomentar la integración, reducir la vulnerabilidad y evitar el aislamiento del colectivo de personas mayores. Las TIC han penetrado en la mayoría de las actividades humanas, lo que plantea nuevos retos educativos (Gros y Contreras (2006). Con las tecnologías de la información, "las personas adquieren nuevas habilidades y formas de participar y expresarse en una sociedad claramente interconectada" (Galera et al., 2018, p.290). Ante una sociedad cada vez más digital e interconectada se requieren nuevas destrezas, competencias y aptitudes. Su desarrollo y posesión, el denominado empoderamiento digital (Mäkinen, 2006) que fomenta la autonomía y el grado de participación ciudadana, son características cada vez más innatas en las nuevas generaciones, pero que en cambio se convierten en un plus a partir de una mayor edad e incluso se convierten en generadoras de bolsas del llamado analfabetismo digital (Pérez-Calle et al., 2020). Un indicador claro de este fenómeno es que el 53,3\% de la población mayor de 65 nunca ha accedido a internet, frente a más del $90 \%$ de usuarios habituales entre jóvenes de 16 a 24 años. Para los mayores de 75 años, el porcentaje de usuarios se queda en un 23\% (Instituto Nacional de Estadística, s.f.; Unión Democrática de Pensionistas y Jubilados de España, 2019). Respecto a las habilidades digitales, tan solo el 6,5\% de los mayores de 65 años cuentan con las destrezas necesarias para el uso de las nuevas tecnologías, con apenas la mitad de las personas de entre 65 y 74 años que, siendo usuarias de internet, se consideran dotadas de las habilidades digitales necesarias para su manejo- y esto a pesar del uso extendido del teléfono móvil desde hace años en esta franja de edad. Estos datos resaltan la existencia de una doble brecha: la primera de ellas, la del acceso a las TIC, en retroceso; la segunda, la de la capacitación para su uso- tanto funcional como actitudinal- sustentado en la falta de motivación, conocimientos y hábitos y que es un hecho que se mantiene en el tiempo (Neves et al., 2013). Además, se puede considerar una tercera brecha digital- implícita en el uso de las nuevas tecnologías y entornos generados- asociada a su capacidad relacional. Esta capacidad supone la evolución y el paso desde las TIC a las denominadas TRIC, término acuñado por Marta-Lazo \& Gabelas (Tecnologías de la Relación, Información y Comunicación), con la inclusión del llamado factor R-elacional, que 
lleva a que las competencias digitales adquieran una nueva dimensión, superando las meras tecnológicas e instrumentales (Gabelas et al., 2015; Marta-Lazo y Gabelas, 2016).

No obstante, si hay un aspecto que en los distintos estudios disponibles merece una menor atención es el relativo a la capacidad para realizar y conseguir la información necesaria para cada necesidad. Esto incluye un grupo de actividades realmente importantes para el desarrollo de la vida diaria de las personas mayores. Se pueden incluir en esta categoría las gestiones bancarias, las gestiones y reclamaciones ante organismos públicos, las denuncias o reclamaciones por compra, los trámites sanitarios, solicitar ayuda a la dependencia, solicitar servicio de ayuda a domicilio sea este teleasistencia o plaza en residencia o denunciar una agresión o maltrato. A este respecto el dato más reseñable ofrecido por los estudios anteriores es que el $60 \%$ no es capaz de realizar trámites por internet. Además, la pandemia de la Covid-19 ha empeorado esta situación de vulnerabilidad, al imposibilitar la realización de diversos trámites por medios no telemáticos (Fernández y Cama, 2020).

La administración, como prestadora única de diversos servicios dirigidos a las personas de más edad, es el principal interlocutor en estas comunicaciones. Ante la necesidad de un mayor apoyo y soporte en sus actividades habituales, los departamentos, consejerías, agencias u oficinas de Salud, Servicios Sociales y Dependencia se enfrentan a la obligación de desarrollar acciones de adaptación y de explicación de los distintos servicios prestados, cuyo contenido debe ser comprendido por las personas en la franja de la tercera edad a las que van dirigidos.

En este punto, entra en juego tanto la necesidad de favorecer y adaptar las nuevas tecnologías a todos los grupos de usuarios como la educación y capacitación en las mismas en un entorno de educación no formal. En este contexto, la OMS plantea el enfoque de la educación para la salud en su documento "Habilidades para la vida" $(\mathrm{HpV})$ : existe una necesidad imperiosa de integrar en la educación aspectos no académicos que respondan no solo a una dimensión cognitiva. Es decir, que también aprendan a ser, a convivir, a hacer y a aprender. De esta forma, el adquirir la destreza en el manejo de las tecnologías de la información y la comunicación tiene un doble efecto. Por un lado, convertirse en receptor de información de forma incomparablemente más rápida, amplia y eficaz $\mathrm{y}$, por otro, incorporarse plenamente a una forma de gestionar todo tipo de asuntos y de procurarse servicios desconocidos hasta ahora, convertirse en un elemento activo y autónomo de una sociedad que vive un cambio radical (Pérez-Calle et al., 2020). Y, en el caso de las personas mayores, facilitar su acceso a las TIC permite que dichas tecnologías sirvan para promocionar la vida independiente, el envejecimiento activo, así como incrementar su participación social.

Distintas administraciones vienen realizando esfuerzos para fomentar la incorporación de las personas mayores a la sociedad digital, creando entornos 
Bunbury Bustillo, E., Pérez Calle, R., y Osuna-Acedo, S.

favorables para este colectivo y suprimiendo barreras que pueden implicar o agravar su aislamiento social. Eliminando la amenaza que supone para ellos las nuevas tecnologías y convirtiéndolas en su lugar en un poderoso aliado en su día a día, en línea con las ventajas potenciales del denominado como envejecimiento activo (Havighurst, 1963).

Así, el Ayuntamiento de Zaragoza, a partir de su adhesión a la Red de Ciudades y Comunidades Amigables con las Personas Mayores de la OMS en 2011 y en el marco de su Plan de Acción, ha puesto en marcha algunas iniciativas para mejorar las competencias digitales de las personas mayores. Entre ellas, dos experiencias que han servido de fuente para el desarrollo de la presente investigación:

- Evaluación de usabilidad de su página web por parte de las propias personas mayores.

- Generación de mapas geolocalizados mediante un proceso co-creativo con las personas mayores de la ciudad.

El presente artículo se organiza como sigue. En primer lugar, tras la introducción al trabajo, se presentan los principales objetivos de la investigación realizada. A continuación, se describe la metodología empleada en la investigación. Posteriormente se presentan los resultados obtenidos, realizando un análisis y discusión de los mismos, para finalizar exponiendo las principales conclusiones extraídas del estudio y la propuesta de próximos pasos a realizar en línea con la investigación.

\section{OBJETIVOS}

Los principales objetivos perseguidos en la presente investigación han sido los siguientes:

- Evaluar si los resultados de esta investigación advierten de una potencial discriminación en competencias digitales.

- Mostrar las ventajas que la superación de la brecha digital podría suponer para los colectivos vulnerables y en concreto para las personas mayores, así como las oportunidades que la capacitación digital brindaría a dicho colectivo.

- Exponer los casos prácticos de dos proyectos reales llevados a cabo en este contexto. 
Bunbury Bustillo, E., Pérez Calle, R., y Osuna-Acedo, S.

Las Competencias Digitales en personas mayores: de amenaza a oportunidad

\section{METODOLOGÍA}

La investigación se ha llevado a cabo mediante el desarrollo y análisis de dos procesos participativos de co-creación (Osuna-Acedo Marta-Lazo \& Frau Meigs, 2018) con personas mayores haciendo uso de las TRIC. Se trata de dos iniciativas que forman parte del programa de Ciudades Amigables con las Personas Mayores del Ayuntamiento de Zaragoza. Por un lado, la elaboración de unas rutas amigables en tres distritos de la ciudad, con sus correspondientes mapas colaborativos y de otra, la evaluación de usabilidad de la web municipal en su apartado de personas mayores.

El primer proceso de co-creación produjo un servicio (mapas colaborativos), que mejora la adecuación de los barrios a la edad a través de rutas adaptadas a la edad y que requiere de acceso a Internet o a dispositivos móviles. El segundo proceso de cocreación produjo una página web en el sitio web municipal que es utilizable y accesible para las personas mayores y contiene toda la información que necesitan.

\subsection{Primer proyecto: Rutas Amigables con las Personas Mayore}

La primera de las iniciativas, Rutas Amigables con las Personas Mayores, fue un proyecto que se puso en marcha con el objetivo de mejorar las rutas más transitadas por personas mayores, a través de su propia participación sugiriendo mejoras y registrándolas en mapas colaborativos de la web del Ayuntamiento de Zaragoza.

Así, una "ruta amigable" es aquella que:

- Es una ruta útil y frecuente: se trata de un recorrido habitual que las personas mayores utilizan en su vida cotidiana en el barrio. Se toma como punto de referencia el Centro de Convivencia del distrito.

- Puede recorrerse andando de manera segura y accesible. Ello implica que las personas mayores habrán estudiado previamente la existencia o no de una serie de necesidades importantes para este colectivo.

- Está elaborada mediante la participación y consenso de un equipo de personas mayores.

Para llevar a cabo el diseño de una ruta y establecer las mejoras para cumplir con los requisitos que la definan como ruta amigable, se desarrolla un proceso basado en la participación y el acuerdo, mediante una metodología que impulsa la inteligencia colectiva a través del consenso intelectual de un grupo, buscando la suma de criterios a la hora de establecer las mejoras para poder definir las rutas amigables. Este fue el diseño metodológico de partida, con el objetivo de validarlo proponiendo mejoras que facilitaran su desarrollo en el futuro.

Las personas mayores fueron los agentes principales del proyecto, su participación fue fundamental para la elaboración de las rutas, por lo que la metodología planteada era plenamente participativa, basada en el consenso y la suma de conocimientos y opiniones, posibilitando la diversidad en la composición de los participantes. 
Respecto a los equipos de personas mayores, cada grupo estuvo formado por 6-8 personas. Para que fueran representativos se procuró que hubiese paridad en cuanto a género y estuvieron formados por mayores de 60 a 75 años y de 76 años en adelante. En cada grupo hubo al menos dos personas con problema físico leve, una persona que le gustase andar o que perteneciera a grupos de senderismo, una persona que fuese usuario/a avanzado de TRIC y dos con un nivel medio. El Ayuntamiento de Zaragoza- a través de su personal técnico- fue quien realizó la selección de los equipos.

Se distribuyeron las tareas que cada persona mayor que formó parte del grupo debía realizar en las sesiones, con el fin de que la rutas más frecuentadas y útiles adquirieran el grado de rutas amigables. Se utilizaron técnicas de dinamización, desarrollando al máximo la participación y creando un ambiente de compañerismo y confianza. Se potenció el trabajo en equipo, la participación y la toma de decisiones, llegando al consenso por acuerdos entre los equipos, utilizando técnicas específicas para ello.

La planificación del proyecto se realizó en base a que todo el proceso debía seguir la metodología de adecuarse y adaptarse tanto a las necesidades y objetivos del proyecto como a las características de los grupos. Así, fue necesario definir los tiempos necesarios a utilizar en cada una de las sesiones, además del número de sesiones a desarrollar. Con el fin de diseñar dos rutas amigables por grupo, se realizó una selección por consenso de los destinos más frecuentes del distrito. Se realizó posteriormente la inclusión de la ruta amigable en el Portal de Gobierno Abierto haciendo uso de la herramienta mapas colaborativos. Las personas dinamizadoras diseñaron y elaboraron los documentos a trabajar con los distintos equipos de personas mayores, recogiendo los siguientes puntos:

- Información acerca de las personas participantes.

- Información acerca de los destinos más frecuentados.

- Propuestas de mejora atendiendo a los siguientes criterios: bancos, semáforos con contador, rebajes de acera, dificultades de acceso en las paradas del autobús, puntos de interés (baños públicos, parques de mayores...), cualquier otro aspecto que estime oportuno el equipo de mayores.

- Informes de evaluación.

Respecto a la ejecución del proyecto, se realizaron en primer lugar, para el análisis previo de los recorridos, distintas rutas a pie junto con cada uno de los grupos de personas mayores. En los distintos recorridos se acordaron las mejoras necesarias para hacer de esa ruta una ruta amigable. Una vez cargada la ruta amigable en la Plataforma de Gobierno Abierto- mapas colaborativos- y establecidas las mejoras necesarias para que pueda ser considerada como tal, se recorrió de forma integral de nuevo cada una de las rutas junto con los miembros de los grupos utilizando los mapas colaborativos en los distintos teléfonos móviles y tablets para la revisión de los 
Bunbury Bustillo, E., Pérez Calle, R., y Osuna-Acedo, S.

Las Competencias Digitales en personas mayores: de amenaza a oportunidad

acuerdos tomados sobre las mejoras necesarias, la medición del tiempo medio necesario y el establecimiento de posibles mejoras en los mapas colaborativos. Todas las propuestas de mejora para las rutas fueron alcanzadas por consenso de los distintos grupos de personas mayores.

\begin{tabular}{|c|c|c|c|c|c|c|c|}
\hline RECURSO & DESCRIPCIÓN & PROPUESTA & DIRECCION & COORDENADAX & COORDEN & FOTOT & NOM \\
\hline Escaleras & Escaleras en mal estado y escalón sin altura & Mayor altura en los escalones & Calle de Sigüess 2, 50017 & 41.647 .136 & -0.913209 & & \\
\hline Alcorques & Alcorques vacios & Rellenar los alcorques & $\begin{array}{l}\text { Calle Mosén Andrés vicente } \\
38\end{array}$ & 41.647 .683 & -0.913276 & sil & 2 \\
\hline Baldosas & Baldosas rotas & Arreglar las baldosas & $\begin{array}{l}\text { Calle Monsén Andrós Vicente } \\
38\end{array}$ & 41.647.815 & -0.913327 & Si & 3 \\
\hline Alcorques & Alcorques vacios y en mitad de la acera & Rellenar los alcorques & $\begin{array}{l}\text { Calle Mosén Andres vicente } \\
36\end{array}$ & 41.648 .107 & -0.913313 & si & 4 \\
\hline Rampa & $\begin{array}{l}\text { Rampa mal acabada, falta baldosa en la } \\
\text { terminación (dificultad para sillas) }\end{array}$ & $\begin{array}{l}\text { Hacer un añadido al final de la } \\
\text { rampa }\end{array}$ & Calle de Ciudadela 16 & 41.648 .337 & -0.912510 & Si & 5 \\
\hline Alcorques & Alcorques sin tapar en acera estrecha & Rellenar los alcorques & Calle Andres Vicente 32 & 41.648 .421 & -0.913803 & Si & $\frac{6}{6}$ \\
\hline Baldosas & Baldosas levantadas & Arreglar las baldosas & Calle Escultor Palao36 & 41.648 .725 & -0.915328 & $\mathrm{si}$ & 7 \\
\hline Alcorques & Alcorques vacios & Rellenar los alcorques & Demetrio Galân Bergua 13 & 41.649 .411 & -0.916256 & Si & 9 \\
\hline Farolas & $\begin{array}{l}\text { La altura de los árboles dificulta la visibilidad de } \\
\text { farolas }\end{array}$ & $\begin{array}{l}\text { Mantenimiento de hojas y } \\
\text { ramas, no permitir que crezcan } \\
\text { tanto los árboles, no colocar las } \\
\text { farolas tan cerca de los arboles }\end{array}$ & & 41.650 .701 & -0.916315 & Si & 10 \\
\hline Alcorques & Alcorques muy profindos & $\begin{array}{l}\text { Rellenar los alcorques y } \\
\text { hacerlos menos profundos }\end{array}$ & Avda. de Madrid 277 & 41.650 .786 & -0.915770 & Si & 11 \\
\hline Limpieza & Falta de limpieza; orina, suciedad, basura... & $\begin{array}{l}\text { Más personal de limpieza } / / \\
\text { educación civical/ multas }\end{array}$ & Avda. de Madrid 267 & 41.650 .985 & -0.914831 & Si & $\begin{array}{l}12 y \\
13\end{array}$ \\
\hline Paso de cebra & $\begin{array}{l}\text { Paso de cebra en mal estado, obstaculo } \\
\text { alcantarilla }\end{array}$ & Arreglar el paso de cebra & $\begin{array}{l}\text { Ada de Madrid y Andrbs } \\
\text { Vicente } 1\end{array}$ & 41.651 .039 & -0.914670 & Si & 14 \\
\hline Marquesina & Ocupa más de la mitad de la acera & & Avenida de Madrid 261 & 41.651 .085 & -0.914143 & Si & 16 \\
\hline Baldosas & Baldosas sueltas y hundidas & Arreglar baldosas & Avenida de Madrid 247 & 41.651 .329 & -0.912763 & SI & 17 \\
\hline Suciedad & Suciedad y excrementos & $\begin{array}{l}\text { Mayor limpieza y medidas para } \\
\text { los duenios de los perros }\end{array}$ & Avda. de Madrid 235 & 41.651 .641 & -0.912108 & Si & 18 \\
\hline Paso de cebra & Rebaje colocado hacia la carretera & Rebaje en el centro & $\begin{array}{l}\text { Avda de Madrid y Cale Sta } \\
\text { Teresita } 1\end{array}$ & 41.651 .756 & -0.911349 & Si & 19 \\
\hline Escaleras & Sucias, en mal estado & Cuidar la limpieza & Avda. Madrid 181 & 41.652 .639 & -0.907404 & Si & $\begin{array}{l}20,2 \\
1,22\end{array}$ \\
\hline Rebajes & No hay rebaje en el paso de peatones & Poner rebaje & Calle Daroca 2 & 41.649 .483 & -0.904459 & Si & 23 \\
\hline Papeleras & Solo 2 papeleras en toda la calle & Poner más papolaras & Calle Daroca & 41.649 .430 & -0.906969 & Si & 24 \\
\hline Paso de cebra & Paso de cebra en mal estado & $\begin{array}{l}\text { Mejorar el mantenirmiento del } \\
\text { paso de cebra }\end{array}$ & Via Universitas 55 & 41.649 .498 & -0.909255 & Si & 25 \\
\hline Paso de cebra & Sobra semáforo y paso de cebra & $\begin{array}{l}\text { dos calles unidas por un único } \\
\text { paso }\end{array}$ & Via Universitas 52-56 & 41.649 .692 & -0.909212 & Si & 26 \\
\hline Alcorques & Agujero en alcorque & Rellenar alcorque & Via Univérsitas 46 & 41.648 .785 & -0.909002 & $\mathrm{Si}$ & 27 \\
\hline Graffitis & Graffitis en bancos $y$ paredes & Multas & $\begin{array}{l}\text { Calle Duquesa Villahermosa } \\
661\end{array}$ & 41.647 .392 & -0.909369 & Si & $\begin{array}{l}26- \\
29\end{array}$ \\
\hline Baldosas & Baldosas sueltas y salpican & Arreglar las baldosas & Duquesa Villahermosa662 & 41.647 .083 & -0.912324 & Si & 30 \\
\hline
\end{tabular}

Figura 1: Ejemplo de la información introducida para la elaboración del mapa colaborativo. Fuente: Ayuntamiento de Zaragoza

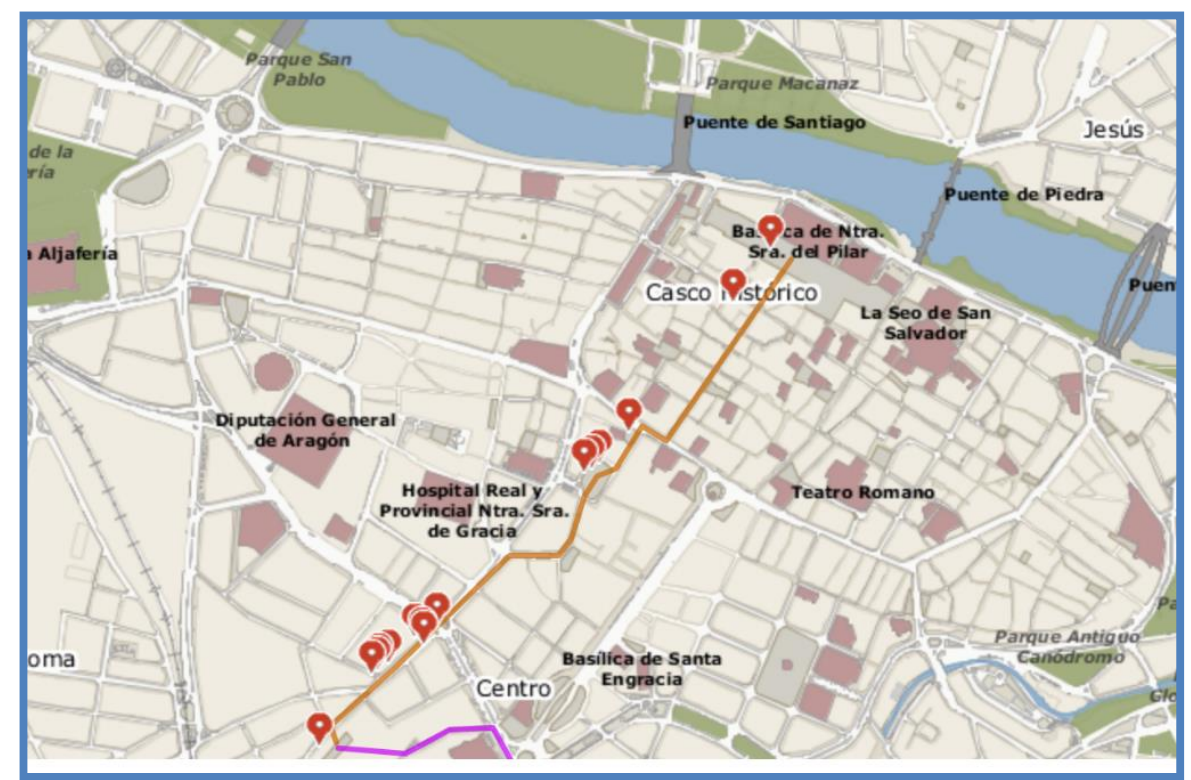

Figura 2: Ejemplo de ruta amigable marcada en los mapas colaborativos de la web del Ayuntamiento de Zaragoza

Fuente: Ayuntamiento de Zaragoza 
Bunbury Bustillo, E., Pérez Calle, R., y Osuna-Acedo, S.

Las Competencias Digitales en personas mayores: de amenaza a oportunidad

\subsection{Segundo proyecto: Mejora de usabilidad web}

La segunda de las iniciativas fue la mejora de usabilidad de una página web dirigida a personas mayores de la web del Ayuntamiento de Zaragoza. Un objetivo que se cumplió en su totalidad ya que todas las conclusiones adoptadas tras el análisis y evaluación de la página web fueron adoptadas. La metodología base del proyecto fue la utilización de grupos focales.

Para llevar a cabo el reclutamiento de los componentes de los grupos se seleccionaron, en diferentes Centros de Convivencia de Personas Mayores, a 85 personas mayores de 65 años, de los que 45 fueron mujeres y 40, hombres. No se conocían entre sí. Los directivos de los diferentes centros se ocuparon de la selección de participantes mediante entrevistas personales, tratando principalmente de averiguar su nivel de conocimiento de nuevas tecnologías.

Respecto al perfil de los participantes, el 53\% eran mujeres y el $47 \%$ hombres. El $47 \%$ se encontraban entre los 65-70 años de edad, el 29\% entre 71 y 75 años de edad y el $24 \%$ mayores de 75 años de edad. El 42\% de las personas participantes habían terminado la educación primaria, el 26\% la educación secundaria, un 13\% poseía estudios universitarios medios y un $15 \%$ estudios universitarios superiores. El $76 \%$ afirmaba no tener ninguna limitación, un 15\% manifestaba tener algún tipo de limitación y un $8 \%$ señaló sí tener limitaciones. En cuanto al uso de nuevas tecnologías, el 25\% manifestaba la utilización de tablet y el móvil, un 34\% utilizaba tablet, ordenador y móvil y el 40\% manifestaba utilizar sólo el móvil.

Siguiendo las recomendaciones de tamaño de entre 6 y 10 personas para llevar a cabo un grupo focal, se dividieron los 85 participantes en ocho grupos homogéneos en función de su experiencia con las nuevas tecnologías. Se realizaron sesiones únicas con cada uno de los grupos, de una duración de 2 horas aproximadamente, en días diferentes. En cada uno de los grupos focales se procuró adaptarse a las características de las personas participantes. Para iniciar cada grupo focal, además de dar la bienvenida a todos los asistentes y seguir las normas habituales de cortesía, se especificó el asunto a tratar con una pequeña charla en la que se establecieron las normas básicas de respeto y de procedimiento en la discusión. A los participantes se les suministró un ordenador a cada uno en el que se mostraba la página web a analizar. La división de los grupos fue la siguiente:

- 4 grupos compuesto por 46 personas con poca o ninguna experiencia (Nivel A) en el ámbito de las nuevas tecnologías. Se dedicó parte de la sesión (entre 30 y 60 minutos) a ofrecerles la información necesaria para utilizar el portal web, es decir, utilización del ratón y barra scroll (barra de desplazamiento lateral), explicación de lo que es una página web y cómo utilizarla. El resto de la sesión se utilizó para realizar las preguntas más básicas de las secciones a analizar (accesibilidad, identidad, arquitectura de la información, navegación y contenido). 
Bunbury Bustillo, E., Pérez Calle, R., y Osuna-Acedo, S.

Las Competencias Digitales en personas mayores: de amenaza a oportunidad

- 2 grupos compuesto por 21 personas con nivel medio (Nivel B) de conocimiento sobre nuevas tecnologías: Se dedicaron unos 30 minutos aproximadamente a preguntarles acerca del uso de otras páginas web, con el objetivo de conocer el nivel, y además establecer comparaciones con el portal de mayores a evaluar.

- 2 grupos compuestos por 18 personas con nivel alto (Nivel C) de conocimiento sobre nuevas tecnologías. Se dedicaron 30 minutos a determinar tanto su nivel de conocimiento de páginas web y a compartir su experiencia con otros usuarios, ya que en muchos casos se trataba de personas mayores voluntarias que forman o apoyan a otras personas mayores en la iniciación de las nuevas tecnologías.

El proceso se desarrolló en todos los casos siguiendo la metodología estipulada para el desarrollo de grupos focales (Callejo, 2001; Wilkinson, 1998), adecuándolo a las características concretas del objeto del estudio y del perfil de los participantes. Además de la realización de un cuestionario específico para preguntar de forma ordenada a los participantes, parte del proceso metodológico se centró en interpelar directamente a las personas para promover la reflexión personal y colectiva que diera también paso a debates atendiendo a las características de los diferentes grupos. Se siguieron las pautas establecidas para generar un ambiente cordial en el que todo el mundo se sintiera cómodo y desinhibido para expresar sus opiniones. Se puso especial atención en evitar la proliferación de opiniones dominantes que invalidaran al resto. Todas las intervenciones fueron grabadas desde el inicio hasta el final de las sesiones.

Con el fin de que el análisis fuese uniforme en todos los grupos se realizaron las mismas preguntas, en una sola batería, adecuando el lenguaje a cada nivel (A, B y C). Como excepción, las personas participantes en el nivel A respondieron a un número inferior de preguntas, más generales, de formulación sencilla y de fácil comprensión. Las personas participantes en el nivel B respondieron a la mayoría de las preguntas y compararon la valoración con otras páginas web que conocían. Para las personas participantes en el nivel C se procuró adecuar las preguntas a su experiencia y nivel, valorando su opinión como usuarios, pero también como formadores de otras personas mayores, lo que permitió conocer su opinión basada en su experiencia con ellas. Para asumir estas opiniones como criterios válidos a tener en cuenta, se valoró la coincidencia de los comentarios en los diferentes grupos, es decir, las opiniones sobre un aspecto en concreto que fueron repetidas por varias personas, así como los puntos de vista personales pero que serían adoptados por los demás, tras el pertinente debate, antes de finalizar la sesión.

Las áreas a evaluar de la página web se dividieron en seis bloques diferentes:

- Accesibilidad

- Identidad 
Bunbury Bustillo, E., Pérez Calle, R., y Osuna-Acedo, S.

- Arquitectura de la información

- Navegación

- Contenidos

Accesibilidad: se valoraron las opiniones y percepciones de los participantes que condicionan la accesibilidad como son el contraste, la imagen, los colores... teniendo en cuenta, además, las posibles limitaciones que pudieran existir de tipo visuales, motrices, auditivas y cognitivas.

Identidad: se valoró la representación gráfica de todos los logos aparecidos en la web, con el fin de comprobar que existe una unidad en la composición en todos los niveles, así como el estilo de la comunicación escrita y la funcionalidad.

Arquitectura de la información: en este apartado se valoró la estructura, organización y etiquetación de los elementos que conforman los entornos informacionales que facilitan la localización- o el acceso- de la información y su utilidad y su aprovechamiento por parte de los usuarios/as.

Navegación: se analizó el orden y agrupación de los contenidos conforme a una categoría que permita al usuario/a identificar las relaciones de contenido, habilitar y facilitar la navegación entre ellos y orientar al usuario.

Contenido: se exploró la coherencia, organización y comprensión de la información principal.

Usabilidad: es un atributo clave de calidad que evalúa el grado de facilidad con el que los usuarios acceden e interactúan con una página web (Nielsen, 2000). Se analizó mediante la experiencia en tiempo real el nivel de usabilidad percibido en los distintos grupos.

\section{DISCUSIÓN}

En la primera de las iniciativas se realizó una evaluación mediante cuestionarios y puesta en común de los equipos del diseño metodológico del que se partía. Los resultados se resumen en los siguientes puntos.

- Equipo de personas mayores: Adecuado en número y composición. El reparto de tareas no supuso mayores dificultades.

- Técnicas de dinamización utilizadas:

- «Presentación por Parejas»: Es necesario que las dinamizadoras cuenten con información previa en cuanto al equipo, ya que si hay varias personas que ya se conocen entre sí, habría o bien, que 
Bunbury Bustillo, E., Pérez Calle, R., y Osuna-Acedo, S.

emparejarlas con personas desconocidas o bien, diseñar otra dinámica que se ajuste mejor. El documento diseñado para la recogida de información sobre los participantes («Perfil del Participante»), ha resultado útil y adecuado.

○ «Reflexión en grupo»: El ejercicio no sólo resultó gratificante para el equipo por la posibilidad de expresar sus experiencias, sino que además arroja información acerca del grado de entendimiento de la tarea que tenían que realizar.

○ «Diagrama de Afinidad»: Es una herramienta de dinámicas grupales que sintetiza un conjunto de datos verbales (destinos frecuentes) agrupándolas en función de la relación que tienen entre sí. Se basa en el principio de que muchos de estos datos son afines, por lo que pueden reunirse, bajo unas pocas ideas generales (rutas cortas, largas - importancia - frecuencia). La dinámica ha resultado totalmente apropiada para el fin que se perseguía.

○ «Sesión de evaluación»: En general, se han mostrado satisfechas. Únicamente, valorarían la posibilidad de aumentar el tiempo en algunas de las sesiones. Se considera absolutamente necesario mantener este ejercicio de evaluación, ya que su opinión es fundamental para diseñar adecuadamente futuras ediciones.

- Planificación: El número de sesiones llevadas a cabo es suficiente para alcanzar los objetivos propuestos; no obstante, de cara a futuras ediciones, se propone realizar una sesión más (entre la Sesión I y II). En cuanto a la duración, se propone aumentar cada sesión de 1,30 horas a 2,00 horas. El horario $(09,30-11,00)$ también resultó adecuado.

- Ejecución: Todo el proceso ha resultado adecuado sin presentar mayor dificultad que el tiempo disponible para ello. Para ediciones futuras, se propone dividir al equipo en dos, de manera que pueda optimizarse el tiempo dedicado a ello.

- Acuerdos por consenso: Todas las decisiones se han tomado mediante consenso. En algunos casos ha sido necesaria la utilización de técnicas diseñadas para ello («Diagrama de Afinidad») que han sido descritas anteriormente, pero para el resto de decisiones se ha utilizado el diálogo y debate en el aula, mediante la participación de todos los componentes del grupo.

La creación de grupos más allá de ajustarse a los perfiles anteriores, no ha generado ningún problema en ninguno de los 3 distritos. Esto no sólo contribuye al carácter participativo del colectivo de personas mayores, sino también a la disponibilidad de una red de centros de mayores donde colaboran y acuden regularmente. A partir de la selección de los participantes, la creación de un grupo 
capaz de trabajar de forma coordinada se ve facilitada, por un lado, por la existencia de un facilitador, que promueve esta dinámica, así como por la propia experiencia de los mayores en el trabajo con otros grupos de personas. La facilidad para conseguir estos dos primeros aspectos en el proceso de co-creación permite un proceso ágil que, como se ha mencionado en otras ocasiones, cuenta con instrumentos y herramientas técnicas que, sin excesiva complejidad, han demostrado ser útiles y eficaces para promover estos procesos de co-creación. La transferencia de estos instrumentos, herramientas y técnicas a otras personas o facilitadores de grupos no representa, por su sencillez, un nivel de dificultad excesivo.

La metodología propia del proyecto- basada en la estimulación de la participación y la toma de acuerdos por consenso, uniendo la experiencia y el conocimiento de los participantes del entorno físico en el que se desarrolla la actividad-ofrece un estímulo permanente en el que todos los participantes implicados en todo el proceso tuvieron en todos los términos.

Entendiendo que las decisiones tomadas se basaron en el acuerdo del grupo, añadiendo además una valoración técnica sobre las mismas, se asume que las decisiones más importantes tomadas- como las de las mejoras a proponer- son adecuadas no sólo a los objetivos del proyecto, en cuanto a estimular la participación y la co-creación, sino también en cuanto a la mejora del entorno urbano.

Con respecto a la segunda de las iniciativas, la evaluación de usabilidad de la página web, se recogieron los siguientes resultados:

\section{Utilización del acceso a internet:}

- Las personas mayores en general disponen de mucho tiempo para dedicarlos a las TIC y sacar provecho de ello, si dispusieran de una formación y capacitación adecuada.

- El uso de ordenadores y de la red Internet no se muestran como cuestiones prioritarias pero cada vez les cuesta menos acercarse a estas tecnologías de la información, ya que cuando superan las barreras iniciales tienden a desarrollar gran interés ante las posibilidades que se les ofrece, lo que facilita su paulatino entusiasmo por el uso de esta herramienta.

- Resultó de gran interés para las personas mayores cada pequeño avance. Agradecieron que se contase con su opinión en algo que les afecta directamente y poder interactuar en la página web.

Usabilidad de la página web:

- Las personas mayores no rechazan la incorporación de tecnología. 
- Las personas mayores son capaces de aprender a usar la tecnología.

- Los aspectos de diseño juegan un papel más importante que la facilidad de uso.

- La barra de scroll les desconcierta. Les cuesta intuir que hay más información en una página que la que se ve en ese momento en la pantalla y que han de usar la barra de scroll para visualizarla.

- Tienden a pinchar sobre una imagen, no sobre el texto subrayado debajo de la misma.

- A las personas principiantes mayores la lupa les induce a pensar que es una herramienta para aumentar el tamaño.

- Los menús desplegables son difíciles de utilizar.

- Los iconos o imágenes relacionadas con un texto en concreto les resultan útiles

- Aunque no era objeto de este estudio, se consideró de interés preguntar a los mayores por el uso de redes sociales (Facebook, Twitter, Instagram). La mayor parte de las personas participantes en los grupos focales no eran usuarios de redes sociales, y además tenían la percepción de que eran muy "peligrosas".

La inclusión de proyectos de este tipo en un plan de acción destinado a mejorar la "amigabilidad" de la ciudad en todos sus aspectos es una importante motivación inicial para los participantes mayores. La producción concreta de mejoras que luego trascienden al ámbito de la gestión pública que se presta a las personas mayores exclusivamente, como por ejemplo la difusión de estas rutas en la página web, la implicación de los servicios locales de parques y jardines y de urbanismo y la inclusión de sus propuestas a través de las juntas de representantes en procesos como los presupuestos participativos, contribuyen definitivamente a la relevancia de este proceso para los participantes.

Teniendo en cuenta que las innovaciones tecnológicas suponen facilitar el acceso a la ciudadanía en diferentes tipos de servicios, sin duda están promocionando la participación social. La creación de una ciudad segura y accesible para las personas mayores les permite permanecer en sus comunidades y barrios $\mathrm{y}$, además, conectarse e interactuar con otros habitantes y formar parte de una "red de seguridad" y contar con información sobre la ubicación de recursos específicos.

La información sobre los recursos en el vecindario, parece ser un buen punto de partida adecuado para involucrar a la ciudadanía mayor en la co-creación de servicios digitales que satisfagan sus necesidades y que ofrezcan gratificaciones a un grupo más amplio de personas mayores. 
Bunbury Bustillo, E., Pérez Calle, R., y Osuna-Acedo, S.

Las Competencias Digitales en personas mayores: de amenaza a oportunidad

\section{CONCLUSIONES}

Con la presentación de estas dos experiencias se ha pretendido evaluar la participación de las personas mayores en el aprendizaje de TRIC, y se ha puesto de manifiesto que son el grupo de mayor desventaja a la hora de obtener y seleccionar información veraz. Y, además, según las experiencias descritas, cuanto mayor es la persona, mayor la brecha que existe.

Las personas que no tienen acceso a las TRIC no sólo están en desventaja ante la información, también lo están ante las posibilidades de ocio y de relación social que las TRIC permiten: vídeollamadas, redes sociales, etc.

Los recursos actuales sobre TRIC, que se iniciaron en el manejo de ordenadores para uso de internet, ofimática, fotografía, etc., progresivamente se ha ido derivando hacia el uso de los móviles y de multitud de aplicaciones que éstos ofrecen, por ser la herramienta más disponible para las personas mayores, pero sobre la que tienen usos bastante limitados. En este sentido, el acceso a las redes sociales es todavía muy limitado, el móvil se usa para la comunicación con familias y amistades, como teléfono y como medio de mensajería a través de Whatsapp.

El manejo de las TRIC ofrece múltiples oportunidades a las personas mayores, que en su mayoría no están explotando: comida a domicilio, compras online, relación no sólo con sus contactos más cercanos, sino también con personas de todo el mundo. Ocupar el tiempo libre de forma ordenada, obtener información cierta y actual, etc. La falta de acceso a las mismas les sitúa en situación de desventaja y de vulnerabilidad frente a otros colectivos de menor edad.

Durante el confinamiento y las restricciones por la pandemia de COVID-19 las personas mayores están siendo víctimas del aislamiento, más aún de lo habitual en muchos casos. La falta de acceso a las TRIC genera mayor aislamiento todavía, lo que deriva en angustias, depresiones, deterioros cognitivos y de salud. Todo ello tiene repercusiones en la vida, en las relaciones sociales y en la capacidad para mantenerse informado y relacionado. La no accesibilidad o falta de capacidad en el uso de TRIC impide a las personas mayores acceder a múltiples recursos disponibles de utilidad lúdica, deportiva o de salud, como por ejemplo vídeos de actividad física, juegos para el fortalecimiento de la memoria, etc.

En los grupos focales descritos anteriormente se detectó que las personas mayores que acceden a las TRIC presentan síntomas de un problema de exceso de información. No sólo se trata de acceder, sino de saber qué información necesitan y dónde las pueden conseguir de forma veraz. Las fake news pueden afectar mucho a las personas mayores, con menor capacidad de comprensión digital.

El objetivo que tienen la mayor parte de las personas mayores es tener autonomía y permanecer en el hogar el máximo tiempo posible, mantenerse en su entorno. Como elemento facilitador de dicho objetivo, que ofrece una gran cantidad de oportunidades para el logro de tal fin, es de máxima importancia la adaptación de 
Bunbury Bustillo, E., Pérez Calle, R., y Osuna-Acedo, S.

Las Competencias Digitales en personas mayores: de amenaza a oportunidad

las tecnologías digitales a las personas mayores y no lo contrario, como se está haciendo en la actualidad. En nuestra opinión, esta adaptación es más importante que las algunas medidas sanitarias que se proponen en la actualidad o los adelantos que ofrece la domótica. Es necesario que las TRIC puedan ser manejadas por cualquier persona más allá de limitaciones por criterios físicos o psíquicos. Para ello, es fundamental el desarrollo de sistemas más intuitivos y visuales, tal y como se ha detectado en la evaluación de la usabilidad de la web, donde elementos como el uso de la barra scroll o la lupa no eran indicadores claros para los participantes en los grupos. Sistemas con mejores controles y apoyo de teleasistencia, sistemas de domicilios accesibles y domésticos.

AGRADECIMIENTOS: Este artículo ha sido realizado en la investigación llevada a cabo desde el Grupo de Investigación en Comunicación e Información Digital (GICID), S-115, reconocido por el Gobierno de Aragón y financiado por el Fondo Social Europeo de Desarrollo Regional, y del Grupo de Investigación en Social Media y Educación Mediática, Inclusiva y Ubicua (SMEMIU) de la Universidad de Educación a Distancia (UNED).

Ha sido realizado también con el trabajo de campo realizado en los dos proyectos mencionados con el Ayuntamiento de Zaragoza.

\section{REFERENCIAS}

Arranz, L., Giménez-Llort, L., De Castro, N. M., Baeza, I., y De la Fuente, M. (2009). El aislamiento social durante la vejez empeora el deterioro cognitivo, conductual e inmunitario. Revista Española de Geriatría y Gerontología, 44(3), 137-142. https://doi.org/10.1016/j.regg.2008.12.001

Callejo, J. (2001). El grupo de discusión: introducción a una práctica de investigación. Madrid: Ariel

Caragliu, A., Del Bo, C., y Nijkamp, P. (2011). Smart cities in Europe. Journal of urban technology, 18(2), 65-82. https://doi.org/10.1080/10630732.2011.601117

Díaz, J. P., y García, A. A. (2018). Envejecimiento demográfico y vejez en España. Panorama social, 28, 11-47. https://www.funcas.es/wpcontent/uploads/Migracion/Articulos/FUNCAS_PS/028art02.pdf

Domènech, J. M. (2019). El envejecimiento de la población española y su impacto macroeconómico. Papeles de Economía Española, (161), 100-241. https://www.jubilaciondefuturo.es/recursos/doc/pensiones/20180910/fondodocumental/el-envejecimiento-de-la-poblacion-espanola-y-su-impactomacroeconomico.pdf

Fernández, M. C. S., y Cama, M. Á. D. (2020). Los tiempos del Covid-19 acentúan la brecha digital como brecha social en La Rioja. In Edunovatic 2020. Conference 
Bunbury Bustillo, E., Pérez Calle, R., y Osuna-Acedo, S.

Las Competencias Digitales en personas mayores: de amenaza a oportunidad

Proceedings: 5th Virtual International Conference on Education, Innovation and ICT, December 10-11, 2020 (pp. 1274-1280). REDINE (Red de Investigación e Innovación

https: / / dialnet.unirioja.es/descarga/articulo/7800276.pdf.

Educativa).

Fernández-Ballesteros, R. (2011). Limitaciones y posibilidades de la edad. IMSERSO. Libro Blanco del envejecimiento activo, 113-148.

Fernández-Ballesteros, R. (2011). Positive ageing: Objective, subjective, and combined outcomes. E-Journal of Applied Psychology, 7(1).

Fernández-Ballesteros, R., Zamarrón, M. D., Bravo, M. D. L., Martínez, M. Á. M., del Moral, R. S., López, P. M., y Nicolás, J. D. (2011). Successful ageing: criteria and predictors. Psychology in Spain, 15(1), 94-101.

Fernández del Moral, J. (2012). La tercera y definitiva brecha digital. Telos: Cuadernos de comunicación e innovación, 91, 6-8.

Gabelas, J. A., Marta-Lazo, C., \& González Aldea, P. (2015). El factor relacional en la convergencia mediática: una propuesta emergente. Anàlisi: quaderns de comunicació i cultura, (53), https://doi.org/0020-34. 10.7238/a.v0i53.2509

Galera, C. G., del Olmo Barbero, J., y del Hoyo Hurtado, M. (2018). Alfabetización mediática, públicos vulnerables y empoderamiento ciudadano. In La educación mediática en España: artículos seleccionados (pp. 287-294). Universitas.

Gros, B., y Contreras, D. (2006). La alfabetización digital y el desarrollo de competencias ciudadanas. Revista Iberoamericana de educación, 42(1), 103-125.

Havighurst, R. J. (1963). Successful aging. Processes of aging: Social and psychological perspectives, 1, 299-320.

Instituto Nacional de Estadística (s.f.). Resumen de datos de Personas por sexo, características demográficas y tipo de uso de TIC. Consultado el 1 de abril de 2021. https://www.ine.es/jaxi/Tabla.htm?tpx=39393\&L=0

Mäkinen, M. (2006). Digital empowerment as a process for enhancing citizens' participation. E-learning and Digital Media, 3(3), 381-395. https://doi.org/10.2304/elea.2006.3.3.381

Marta-Lazo, C. y Gabelas, J. A., (2016). Comunicación digital: Un modelo basado en el Factor R-elacional. Editorial UOC.

Ministerio de Sanidad, 2020. Demografía, indicadores de bienestar y determinantes sociales. https://cpage.mpr.gob.es/ 
Bunbury Bustillo, E., Pérez Calle, R., y Osuna-Acedo, S.

Las Competencias Digitales en personas mayores: de amenaza a oportunidad

Neves, B. B., Amaro, F., y Fonseca, J. R. (2013). Coming of (old) age in the digital age: ICT usage and non-usage among older adults. Sociological Research Online, 18(2), 22-35. https:// doi.org/10.5153/sro.2998

Nielsen, J. (2000). Designing Web Usability. Pearson Education

Organización Mundial de la Salud (s.f.). Age-friendly-environments. Consultado el 28 de marzo de 2020. https://www.who.int/ageing/age-friendlyenvironments/en/

Organización Mundial de la Salud. (2007). Ciudades globales amigables con los mayores: una guía. https://www.who.int/ageing/AFCSpanishfinal.pdf?ua=1

O'shea, E. (2003). La mejora de la calidad de vida de las personas mayores dependientes. Galway: Universidad Nacional de Irlanda, 6. http:// envejecimiento.csic.es/documentos/documentos/oshea-mejora-01.pdf

Osorio, A. G., Rosero, M. Y. T., Ladino, A. M. M., Garcés, C. A. V., y Rodríguez, S. P. (2010). La Promoción de la Salud como Estrategia para el Fomento de Estilos de Vida Saludables. Hacia la Promoción de la Salud, 15(1), 128-143.

Osuna-Acedo, S., Marta-Lazo, C. y Frau-Meigs, D. (2018). De sMOOC a tMOOC, el aprendizaje hacia la transferencia profesional: El proyecto europeo ECO. Comunicar: Revista científica iberoamericana de comunicación y educación, 55, 105-114. https://doi.org/10.3916/C55-2018-10

Petretto, D. R., Pili, R., Gaviano, L., López, C. M., y Zuddas, C. (2016). Envejecimiento activo y de éxito o saludable: una breve historia de modelos conceptuales. Revista española de geriatría y Gerontologia, 51(4), 229-241. https://doi.org/10.1016/j.regg.2015.10.003

Pérez-Calle, B., Marta-Lazo, C., y Nogales-Bocio, A. I. (2020). Diseño de un proyecto de capacitación en competencias digitales de los colectivos vulnerables, mediadores en educación permanente y MYPES-PYMES en Aragón.

Puig Llobet, M., Lluch Canut, M. T., y Rodríguez Ávila, N. (2009). Valoración de enfermería: detección de la soledad y del aislamiento social en los ancianos de riesgo. Gerokomos, 20(1), 9-14.

Rodríguez-Porrero, C., y Gil González, S. (2014). Ciudades amigables con la edad, accesibles e inteligentes. Madrid Centro de Referencia Estatal de Autonomía Personal y Ayudas Técnicas-CEAPAT

Telefónica, F. (2011). Smart Cities: un primer paso hacia la Internet de las Cosas (Vol. 16). Ariel.

Triadó, C. y Villar, F. (2008). Envejecer en positivo. Aresta. 
Bunbury Bustillo, E., Pérez Calle, R., y Osuna-Acedo, S.

Las Competencias Digitales en personas mayores: de amenaza a oportunidad

Unión Democrática de Pensionistas y Jubilados de España (2019). Informe sobre indefensión y vulnerabilidad. https://www.mayoresudp.org/wpcontent/uploads/2020/03/20049ISAS01-Informe-de-resultados-NOV-

Vulnerabilidad.pdf

Unión Democrática de Pensionistas y Jubilados de España (2019). Informe sobre brecha digital. https://www.mayoresudp.org/wpcontent/uploads/2019/09/19024-19038-UDP-BM-INF-1-FEB_Brecha-digital.pdf

Wilkinson, S. (1998). Focus group methodology: a review. International journal of social research methodology,

https://doi.org/10.1080/13645579.1998.10846874

Zamarro Cuesta, J. (2017). Red mundial de ciudades y comunidades amigables. Autonomía personal, 19, 47-51.

\section{AUTORES:}

Eva Bunbury Bustillo.

Licenciada en Filosofía y Letras (Filología Inglesa) por la Universidad de Zaragoza, Máster en Comunidades Europeas y Unión Europea por el Real Instituto de Estudios Europeos, Practitioner en Programación Neurolingüiística (PNL). Consultora en Bunbury \& Asociados desde hace de 10 años, una consultoría especializada en la gestión y consecución de proyectos europeos e internacionales, y coach en comunicación. Compagina desde hace 3 años su actividad profesional con la docencia como Profesora Asociada en la Facultad de Ciencias de la Salud de la Universidad de Zaragoza. Es miembro de los grupos de investigación de GICID en dicha universidad, y de SMEMIU, en la UNED. Sus líneas de investigación principales son la programación neurolingüística (PNL) en el aprendizaje de idiomas, y la digitalización en comunicación y enseñanza.

Orcid ID: https://orcid.org/0000-0002-7547-3019

Google Scholar: https://scholar.google.es/citations?hl=es\&user=MIFx7XIAAAAJ 
Bunbury Bustillo, E., Pérez Calle, R., y Osuna-Acedo, S.

Las Competencias Digitales en personas mayores: de amenaza a oportunidad

\section{Ricardo Pérez Calle.}

Ingeniero Industrial por la Universidad de Zaragoza, Master of Business Administration (MBA) por el Instituto de Empresa Business School, Executive MBA por la Universidad Europea. Responsable de Operaciones durante 14 años en empresas industriales, actualmente es consultor de negocio y de proyectos europeos. Compagina, desde 2008, su actividad profesional con la docencia como Profesor Asociado en el Departamento de Dirección y Organización de Empresas de la Universidad de Zaragoza, siendo miembro de los grupos de investigación GICID, en dicha universidad, y de SMEMIU, en la UNED. Sus líneas de investigación principales son la calidad de la gestión organizacional, los determinantes del performance empresarial, la optimización de procesos y la digitalización de las organizaciones.

Orcid ID: https://orcid.org/0000-0003-2726-7146

Google Scholar: https://scholar.google.es/citations?user=36YyA2gAAAAJ\&hl=es

\section{Sara Osuna-Acedo.}

Catedrática de Educación en la Universidad Nacional de Educación a Distancia (UNED). Investigadora Principal del Grupo de Investigación en Social Media y Educación Mediática Inclusiva y Ubicua (SMEMIU). Vicepresidenta de la spin-off tecnológica de la UNED "ECO Digital Learning". Ha escrito más de cien publicaciones, entre libros, capítulos y artículos relacionados con sus líneas de investigación: educación mediática, competencias digitales, sMOOC, tMOOC y usos e interacciones en redes sociales.

Orcid ID: https:/ / orcid.org/0000-0002-5454-6215

\section{Google Scholar:}

https://scholar.google.com/citations?user=Vifi3McAAAAJ\&hl=es\&oi=ao 